\title{
Efficacy and safety of lapatinib in Chinese breast cancer patients: a real-world study
}

\author{
Xuan Ye, ${ }^{1,2 \#}$ Xin Luo ${ }^{1,2 \#}$, Qiong $\mathrm{Du}^{1,2,3 \#}$, Huan $\mathrm{Li}^{1,2}$, Hong-Yue Liu ${ }^{1,2}$, Bo Yu ${ }^{4}$, Qing Zhai ${ }^{1,2}$ \\ ${ }^{1}$ Department of Pharmacy, Fudan University Shanghai Cancer Center, Shanghai 200032, China; ${ }^{2}$ Department of Oncology, Shanghai Medical \\ College, Fudan University, Shanghai 200433, China; ${ }^{3}$ Department of Pharmacy, Minhang District Cancer Hospital, Shanghai 200240, China; \\ ${ }^{4}$ Department of Pharmacy, Tongren Hospital, Shanghai Jiao Tong University School of Medicine, Shanghai 200336, China \\ Contributions: (I) Conception and design: X Ye, B Yu, Q Zhai; (II) Administrative support: Q Zhai, B Yu; (III) Provision of study materials or patients: \\ X Ye, X Luo, Q Du; (IV) Collection and assembly of data: H Li, HY Liu; (V) Data analysis and interpretation: X Ye, X Luo; (VI) Manuscript writing: \\ All authors; (VII) Final approval of manuscript: All authors. \\ "These authors are contributed equally to this work. \\ Correspondence to: Qing Zhai. Department of Pharmacy, Fudan University Shanghai Cancer Center, No. 270 Dong-An Road, Shanghai 200032, \\ China. Email: zhaiqing63@126.com.
}

Background: Lapatinib is approved for the treatment of metastatic HER2-overexpressed breast cancer with capecitabine after progress on anthracycline, taxane, and trastuzumab in China. A post-marketing pharmacovigilance program was carried out to verify the real-world safety and the efficacy information of lapatinib.

Methods: This was a prospective, non-interventional, long-term study in the real-world setting. All patients treated with lapatinib during the program (inclusion period 12 months) in Fudan University Shanghai Cancer Center (FUSCC) were included. The main outcome measures were progression-free survival (PFS) and incidence of adverse events.

Results: A total of 112 patients were enrolled. The median age was 52 years, $64.3 \%$ of patients were postmenopausal, 90 patients (80.4\%) had stage IV disease, and the most common metastatic site was in the lung (43.8\%), bone (30.4\%), liver (26.8\%), and brain (18.8\%). About half of the patients (46.4\%) experienced 3 or more systemic regimens before lapatinib. After a median follow-up of 34.3 months (range, 17.9-57.9 months), the median PFS was 8.1 months (95\% CI, 5.8 to 10.4 months). Later phase of disease (stage IV), 3 or more prior treatments, pulmonary metastasis, liver metastasis, prior anthracycline or taxane, and poor adherence strongly correlated with worse survival $(\mathrm{P}<0.005)$. The grade 3 or 4 adverse events were diarrhea $(9.8 \%)$, hand-foot syndrome (5.4\%), and rash (4.5\%).

Conclusions: Upon implementation of lapatinib therapy in a real-world setting, the case mix was characterized by more early-stage breast cancer patients. The median PFS was slightly superior to what was published in the clinical trials. Pulmonary metastasis or liver metastasis significantly correlated with worse survival. We reported a similar prevalence of adverse events.

Keywords: Lapatinib; Chinese; safety; efficacy; breast cancer

Submitted Feb 12, 2020. Accepted for publication Mar 10, 2020.

doi: 10.21037/atm.2020.03.21

View this article at: http://dx.doi.org/10.21037/atm.2020.03.21

\section{Introduction}

Lapatinib, a HER2 tyrosine kinase inhibitor, is approved for use in combination with capecitabine for the treatment of locally advanced or metastatic HER2-overexpressed breast cancer after progress on anthracycline, taxane, and trastuzumab in China. A superior survival benefit of lapatinib plus capecitabine was demonstrated in one phase 3 trial (1) among HER2-positive advanced breast cancer patients treated, compared to capecitabine monotherapy. 
Although there was no significant difference in overall survival (OS) between the combination therapy and monotherapy groups due to the cross-over design, the addition of lapatinib significantly improved the time to progression (TTP) from 4.4 to 8.4 months. Furthermore, the exploratory analysis confirmed an OS improvement from 64.7 to 75.0 weeks $(\mathrm{P}=0.210)$ (2).

Approval of lapatinib in China was based on data from a single-arm, open-lable study (EGF10949) (3), which was initiated to evaluate the efficacy and safety of lapatinib plus capecitabine in Chinese patients with patients with HER2positive metastatic breast cancer. The results showed that the median PFS was 6.34 months (95\% CI, 4.93-9.82 months) and that the toxicities were well-tolerated.

As information on case mix characteristics that influence the safety and effectiveness of lapatinib therapy is limited for the clinical trial setting, we conducted a post-marketing surveillance program to investigate the safety and effectiveness of lapatinib. Moreover, we wanted to identify those factors that might have significantly affected the clinical outcomes.

\section{Methods}

\section{Study design and population}

This is a prospective, non-interventional, long-term study in the real-world setting. The study was approved by the ethics committee at Fudan University Shanghai Cancer Center (FUSCC). Between July 2016 and June 2017 (an inclusion period of 12 months), all breast cancer patients treated with lapatinib and with informed consent were included.

\section{Collected information}

The Case Report Form (CRF) was filled in via an electronical data capturing system. The main information that was collected included (I) case mix and prior therapy characteristics, (II) efficacy of lapatinib and progressionfree survival (PFS), and (III) incidence of adverse events as per the National Cancer Institute Common Terminology Criteria for Adverse Events (NCI-CTCAE, version 5.0).

\section{Follow-up}

Patients were prospectively followed up at 4,8 , and 24 weeks after the procedure, with adverse events collection at each visit, and lapatinib was prescribed to a limited maximum of 3 cycles. Therefore, patients were followed-up every 1-3 months at our institution or by a general physician. The end of the follow-up was May 2019.

\section{Statistical analysis}

Survival curves were estimated with Kaplan-Meier method. The differences between survivals were estimated using the log-rank test. Multivariate Cox regression analysis was used to evaluate any independent prognostic effect of the variables on PFS. All data were analyzed using IBM SPSS 20.0 for Windows. A P value $<0.05$ was considered significant.

\section{Results}

\section{Patients and treatments}

Between July 2016 and June 2017, a total of 112 breast cancer patients were enrolled in this study. The median age of the patients was 52 years (range, 29-72 years) (Table 1). More than half $(64.3 \%)$ of the patients were postmenopausal, with $80.4 \%$ in stage IV, $17.0 \%$ in stage III, and $2.7 \%$ in stage II. The most common metastatic site was in the lung $(43.8 \%)$, bone (30.4\%), liver $(26.8 \%)$, and brain $(18.8 \%)$.

Nearly all (92.9\%) patients had received at least 1 systemic therapy, and $46.4 \%$ had received 3 or more chemotherapies (Table 2). Only $10.7 \%$ of patients were trastuzumab-naive. When initiating lapatinib treatment, the most common combination drug was capecitabine, paclitaxel, or vinorelbine. The initial dosage of lapatinib was 1,250 and $1,000 \mathrm{mg}$ in $66.1 \%$ and $33.0 \%$ of the patients, respectively. Only 1 patient received $500 \mathrm{mg}$ as a loading dose.

\section{Efficacy}

The median follow-up was 34.3 months (range, 17.957.9 months), and the median PFS was 8.1 months (95\% CI, 5.8-10.4 months) in the total population (Figure 1).

Factor analysis was conducted according to patientrelated, tumor-related, and therapy-related characteristics (Figure 2). Compared to the patients in stage II/III, patients who had stage IV diseases were strongly correlated with worse survival (median PFS, 15.8 vs. 6.2 months; $\mathrm{P}<0.001$ ). The number and site of metastasis also influenced the survival with lapatinib, and fewer metastases predicted a better 
Table 1 Base-line characteristics

\begin{tabular}{|c|c|}
\hline Characteristics & $\mathrm{N}=112$ \\
\hline \multicolumn{2}{|l|}{ Age/year } \\
\hline Median & 52 \\
\hline Range & $29-72$ \\
\hline \multicolumn{2}{|c|}{ Menopausal status, n (\%) } \\
\hline Pre-menopause & $40(35.7)$ \\
\hline Post-menopause & $72(64.3)$ \\
\hline \multicolumn{2}{|c|}{ Hormone receptor, n (\%) } \\
\hline $\mathrm{ER}(+)$ & $47(42.0)$ \\
\hline PR $(+)$ & $33(29.5)$ \\
\hline \multicolumn{2}{|c|}{ Molecular subtyping, $\mathrm{n}(\%)$} \\
\hline Luminal A & $9(8.0)$ \\
\hline Luminal B & $35(31.3)$ \\
\hline $\operatorname{HER}(+)$ & $64(57.1)$ \\
\hline \multicolumn{2}{|l|}{ Disease stage, n (\%) } \\
\hline II & $3(2.7)$ \\
\hline III & $19(17.0)$ \\
\hline IV & $90(80.4)$ \\
\hline \multicolumn{2}{|c|}{ Common metastatic site, $\mathrm{n}(\%)$} \\
\hline Pulmonary & $49(43.8)$ \\
\hline Bone & $34(30.4)$ \\
\hline Liver & $30(26.8)$ \\
\hline Brain & $21(18.8)$ \\
\hline Others & $15(13.4)$ \\
\hline \multicolumn{2}{|c|}{ Number of metastatic sites, $\mathrm{n}(\%)$} \\
\hline 0 & $22(19.6)$ \\
\hline 1 & $49(43.8)$ \\
\hline 2 & $26(23.2)$ \\
\hline$\geq 3$ & $15(13.4)$ \\
\hline
\end{tabular}

survival. There was a median PFS of 11.7 months in patients with no more than 1 metastasis site versus 5.7 months for patients with 2 or more metastasis sites. The presence of pulmonary or liver metastasis significantly reduced the PFS by about 5 months compared to other metastasis sites $(\mathrm{P}<0.001)$. Previous chemotherapy also affected the survival with lapatinib: patients with 3 or more prior systemic
Table 2 Therapy characteristics

\begin{tabular}{lc}
\hline Therapy characteristics & $\mathrm{N}=112$ \\
\hline Prior systemic regimens, $\mathrm{n}(\%)$ & $8(7.1)$ \\
0 & $31(27.7)$ \\
1 & $21(18.8)$ \\
2 & $17(15.2)$ \\
3 & $13(11.6)$ \\
4 & $9(8.0)$ \\
5 & $13(11.6)$ \\
$\geq 6$ & \\
Type of prior systemic regimens, $\mathrm{n}(\%)$ & $79(70.5)$ \\
Anthracycline-based & $102(91.1)$ \\
Taxane-based & $45(40.2)$ \\
Platinum-based & $54(48.2)$ \\
Fluorouracil-based & $38(33.9)$ \\
Endocrinotherapy & $100(89.3)$ \\
Trastuzumab & $78(69.6)$ \\
Combined regimen of lapatinib, $\mathrm{n}(\%)$ & $21(18.8)$ \\
Capecitabine & $76.3)$ \\
Paclitaxel weekly & \\
\hline & \\
\hline & \\
\hline & \\
\hline &
\end{tabular}

therapies tended to experience worse PFS compared to patients with fewer than 3 prior chemotherapies (12.2 vs. $5.7 \mathrm{~m}, \mathrm{P}<0.001)$. In addition, previous treatment with a fluorouracil-, anthracycline-, or taxane-based regimen was found to correlate with worse survival.

\section{Safety}

Adverse drug reactions (ADRs) of all grades and grade $\geq 3$ occurred in 99 patients (88.4\%, 327 events) and 29 patients (25.9\%, 37 events), respectively (Table 3). ADRs (any grade) that were reported in $\geq 10 \%$ of patients included diarrhea (50.9\%), rash (40.2\%), hand-foot syndrome (28.6\%), hepatobiliary events $(17.0 \%)$, paronychia $(15.2 \%)$, and stomatitis $(10.7 \%)$. The most frequent grade $\geq 3$ ADRs were diarrhea (9.8\%), hand foot syndrome (5.4\%), and rash $(4.5 \%)$. 


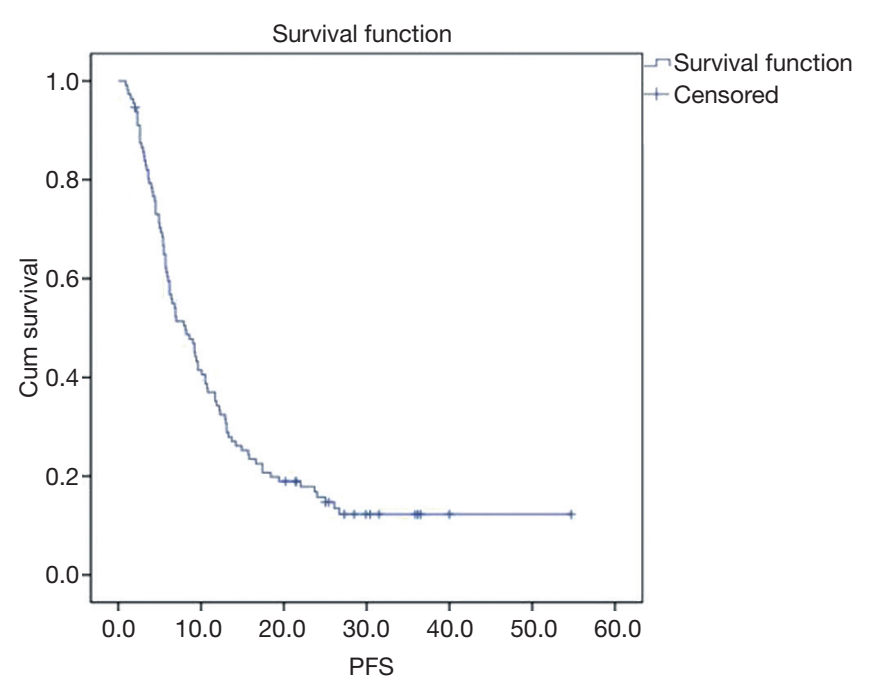

Figure 1 Kaplan-Meier curve for progression-free survival of 112 patients with HER2-positive breast cancer treated with lapatinib therapy. Median PFS was 8.1 months (95\% confidence interval, 5.8-10.4 months).

\section{Discussion}

This study was performed in real-world settings to evaluate the safety and efficacy of lapatinib in Chinese patients with HER2-positive breast cancer. The median PFS (8.1 months) of lapatinib observed in this study was generally superior to those reported in the clinical trial setting $(3,4)$, and a similar prevalence of adverse events was observed.

Regarding efficacy, median PFS in the present study was 8.1 months, which is superior to the median PFS (6.4 months) reported by Xu et al. (3) or that (6.2 months) by Cameron et al. (4). One possible explanation for the differences in PFS is the high proportion of patients (19.7\%) with early-stage breast cancer in our study with only about $1.9 \%$ and $4 \%$ of early-stage patients in EGF109491 and in EGF100151, respectively.

Lapatinib has been approved to be used in combination with capecitabine to treat breast cancer. However, the present study found that, in terms of combination regimen, the off-label use was common. A weekly paclitaxel, vinorelbine, and gemcitabine regimen was usually the off-label combination of choice, with respective rates of $18.8 \%, 6.3 \%$, and $4.5 \%$. Lapatinib plus paclitaxel was evaluated in Japanese metastatic breast cancer women as first-line therapy and demonstrated good tolerance and a median OS of 35.6 months (5). Furthermore, lapatinib plus paclitaxel has been proven to significantly prolong OS and
PFS compared with placebo plus paclitaxel in a phase III, randomized, double-blind study (OS, 27.8 vs. 20.5 months, $\mathrm{P}=0.0124$; PFS, 9.7 vs. 6.5 months, $\mathrm{P}<0.001$ ) (6). Lapatinib combined with capecitabine, vinorelbine, or gemcitabine was directly compared in a phase II randomized trial, suggesting lapatinib plus vinorelbine or gemcitabine seem to be effective and tolerable regimens for HER2positive metastatic breast cancer patients with prior taxane therapy (7). No significant difference in OS was found in a global multicenter phase II trial between capecitabine or vinorelbine groups (8). Although these kinds of combinations have been supported by clinical trials to some extent, none of them have been recommended by an authoritative guideline with high level evidence. However, dual anti-HER2 strategy-lapatinib plus trastuzumab, as a neoadjuvant treatment, appeared in $8.0 \%$ of cases in this research. This modality is consistent with some metaanalyses $(9,10)$ the finding of the NeoALTTO trial $(11)$, and also recommended by the National Comprehensive Cancer Network (NCCN) guidelines (category 2A).

Because of a broader case mix in the real-world setting, we conducted an explorative subgroup analysis to find the affecting factors of lapatinib efficacy. The results showed that later phase of disease (stage IV), 3 or more prior treatments, pulmonary metastasis, liver metastasis, prior anthracycline or taxane therapy, and poor adherence, strongly correlated with worse survival. Interestingly, Cameron et al. (2) reported that liver metastases was a negative prognostic factor of breast cancer patients treated with lapatinib. A retrospective study in India also showed that the presence of liver metastasis when starting lapatinib also correlated strongly with worse survival (12).

The safety data prospectively collected in the current study is similar to those in clinical trials (2-4). The severe adverse events reported in this research included diarrhea, hand-foot syndrome, rash, paronychia, and hepatobiliary events. According to an update on serious adverse events (SAEs), the most common SAE in the combination group was also diarrhea, and some other frequently reported SAEs were dehydration, dyspnea, and vomiting (2). Hepatobiliary events, in particular, have drawn our focus for both a clinical trial emphasis and an existing correlation of liver metastases and prognosis.

\section{Conclusions}

In conclusion, breast cancer patients treated with lapatinib were characterized by more varied conditions, by moderate- 


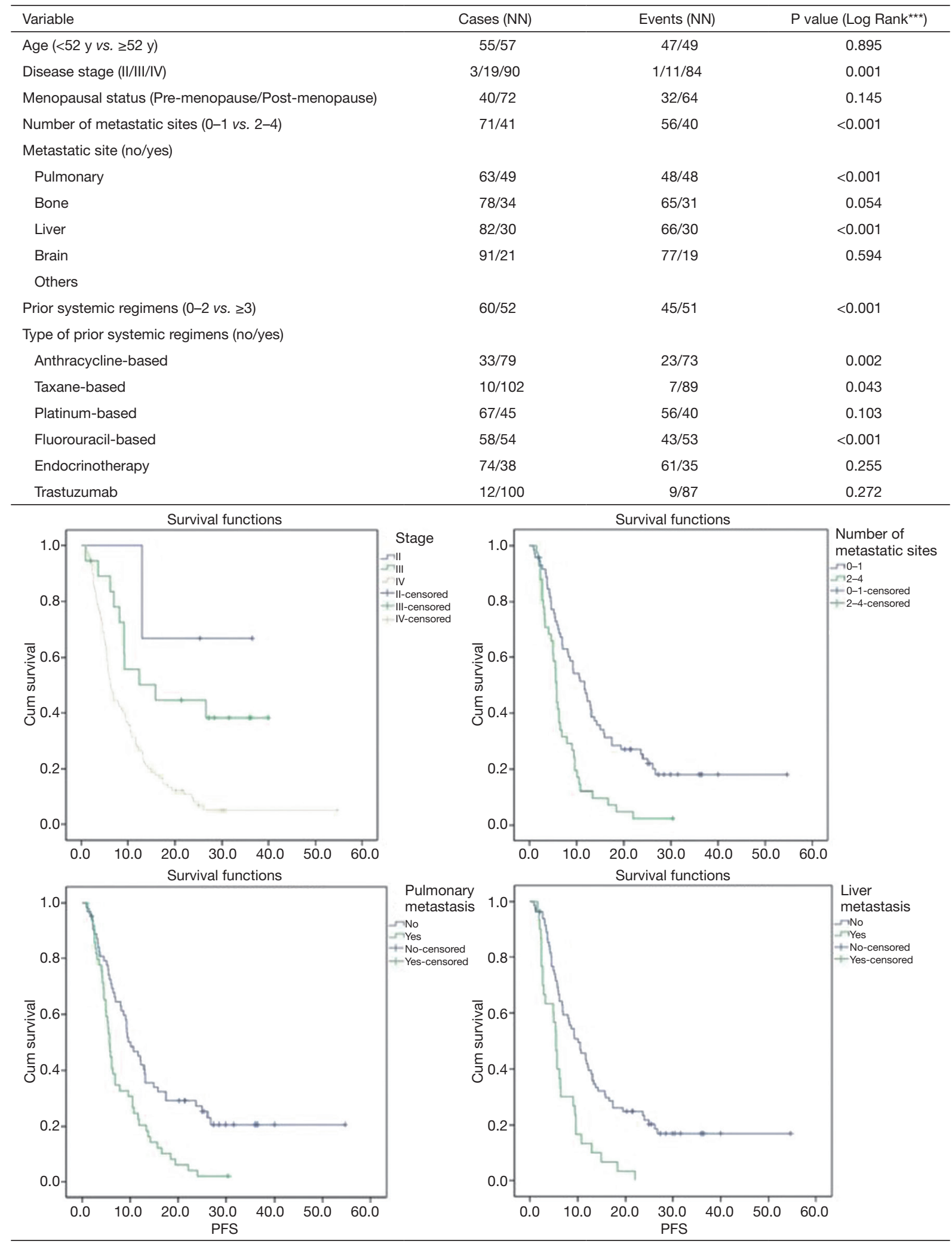

Figure 2 Kaplan-Meier curves for survival in predefined subgroups. ${ }^{* * *}, \mathrm{P}<0.001$. 
Table 3 Main treatment-related adverse events

\begin{tabular}{lcr}
\hline Adverse events & All grades (\%) & Grade 3-4 (\%) \\
\hline Diarrhea & $57(50.9)$ & $11(9.8)$ \\
Rash & $45(40.2)$ & $5(4.5)$ \\
Hand-foot syndrome & $32(28.6)$ & $6(5.4)$ \\
Hepatobiliary events & $19(17.0)$ & $1(0.9)$ \\
Paronychia & $17(15.2)$ & $3(2.7)$ \\
Stomatitis & $12(10.7)$ & $0(0.0)$ \\
\hline
\end{tabular}

to-severe disease, and by light-to-heavy prior therapy. Efficacy and safety information has been compared and verified in trials. Further studies are warranted in the subgroup of pulmonary and liver metastases to determine lapatinib administration in breast cancer patients.

\section{Acknowledgments}

Funding: This study was supported by the Shanghai Municipal Commission of Health and Family Planning (2016ZB0301-02), and funding from the Project for Key Subjects on Clinical Pharmacy was given to Q Zhai.

\section{Footnote}

Conflicts of Interest: The authors have no conflicts of interest to declare.

Ethical Statement: The authors are accountable for all aspects of the work in ensuring that questions related to the accuracy or integrity of any part of the work are appropriately investigated and resolved. The study was approved by the ethics committee at Fudan University Shanghai Cancer Center (FUSCC) (No. 2016-106-1159K1). Between July 2016 and June 2017 (an inclusion period of 12 months), all breast cancer patients treated with lapatinib and with informed consent were included.

Open Access Statement: This is an Open Access article distributed in accordance with the Creative Commons Attribution-NonCommercial-NoDerivs 4.0 International License (CC BY-NC-ND 4.0), which permits the noncommercial replication and distribution of the article with the strict proviso that no changes or edits are made and the original work is properly cited (including links to both the formal publication through the relevant DOI and the license).
See: https://creativecommons.org/licenses/by-nc-nd/4.0/.

\section{References}

1. Geyer CE, Forster J, Lindquist D, et al. Lapatinib plus capecitabine for HER2-positive advanced breast cancer. N Engl J Med 2006;355:2733-43.

2. Cameron D, Casey M, Oliva C, et al. Lapatinib plus capecitabine in women with HER-2-positive advanced breast cancer: final survival analysis of a phase III randomized trial. Oncologist 2010;15:924-34.

3. Xu BH, Jiang ZF, Chua D, et al. Lapatinib plus capecitabine in treating HER2-positive advanced breast cancer: efficacy, safety, and biomarker results from Chinese patients. Chin J Cancer 2011;30:327-35.

4. Cameron D, Casey M, Press M, et al. A phase III randomized comparison of lapatinib plus capecitabine versus capecitabine alone in women with advanced breast cancer that has progressed on trastuzumab: updated efficacy and biomarker analyses. Breast Cancer Res Treat 2008;112:533-43.

5. Inoue K, Kuroi K, Shimizu S, et al. Safety, pharmacokinetics and efficacy findings in an open-label, single-arm study of weekly paclitaxel plus lapatinib as firstline therapy for Japanese women with HER2-positive metastatic breast cancer. Int J Clin Oncol 2015;20:1102-9.

6. Guan Z, Xu B, DeSilvio ML, et al. Randomized trial of lapatinib versus placebo added to paclitaxel in the treatment of human epidermal growth factor receptor 2-overexpressing metastatic breast cancer. J Clin Oncol 2013;31:1947-53.

7. Gómez HL, Neciosup S, Tosello C, et al. A Phase II Randomized Study of Lapatinib Combined With Capecitabine, Vinorelbine, or Gemcitabine in Patients With HER2-Positive Metastatic Breast Cancer With Progression After a Taxane (Latin American Cooperative 
Oncology Group 0801 Study). Clin Breast Cancer 2016;16:38-44.

8. Janni W, Sarosiek T, Karaszewska B, et al. Final overall survival analysis of a phase II trial evaluating vinorelbine and lapatinib in women with ErbB2 overexpressing metastatic breast cancer. Breast 2015;24:769-73.

9. Xu ZQ, Zhang Y, Li N, et al. Efficacy and safety of lapatinib and trastuzumab for HER2-positive breast cancer: a systematic review and meta-analysis of randomised controlled trials. BMJ Open 2017;7:e013053.

10. Xin Y, Guo WW, Huang Q, et al. Effects of lapatinib or trastuzumab, alone and in combination, in human

Cite this article as: Ye X, Luo X, Du Q, Li H, Liu HY, Yu B, Zhai Q. Efficacy and safety of lapatinib in Chinese breast cancer patients: a real-world study. Ann Transl Med 2020;8(5):240. doi: 10.21037/atm.2020.03.21 epidermal growth factor receptor 2-positive breast cancer: a meta-analysis of randomized controlled trials. Cancer Med 2016;5:3454-63.

11. Baselga J, Bradbury I, Eidtmann H, et al. Lapatinib with trastuzumab for HER2-positive early breast cancer (NeoALTTO): a randomised, open-label, multicentre, phase 3 trial. Lancet 2012;379:633-40.

12. Göksu SS, Bozcuk H, Koral L, et al. Factors predicting lapatinib efficacy in HER-2 + metastatic breast carcinoma: Does it work better in different histologic subtypes? Indian J Cancer 2015;52:517-9. 